both physical and cognitive domains, but over time, the FM group also experienced significantly more accelerated rates of decline compared to their healthy counterparts. Findings suggest that chronic pain conditions like FM exacerbate declines in physical and cognitive performance beyond normative aging changes. Understanding issues related to aging with chronic pain may help reduce disability and medical costs, improve physical and cognitive performance, and enhance quality of life.

\section{SARCOPENIA IN PERIPHERAL ARTERIAL DISEASE: PREVALENCE AND IMPACT ON FUNCTIONAL STATUS.}

O. Addison ${ }^{1,2}$, S.J. Prior ${ }^{1,2}$, R. Kundi ${ }^{3}$, A.W. Gardner ${ }^{4}$, A.S. Ryan ${ }^{2,1}, 1$. University of Maryland, Baltimore, Baltimore, Maryland, 2. Department of Veterans Affairs and Veterans Affairs Medical Center Baltimore, Geriatric Research, Education and Clinical Center (GRECC)., Baltimore, Maryland, 3. Department of Surgery, Division of Vascular Surgery, University of Maryland, Baltimore, MD, Baltimore, Maryland, 4. Reynolds Oklahoma Center on Aging, Donald W. Reynolds Department of Geriatric Medicine, University of Oklahoma Health Sciences Center (OUHSC), Oklahoma City, Oklaboma

Peripheral arterial disease (PAD) results in ischemic related pathological changes in the affected muscles. To alleviate exertional leg symptoms, those with PAD decrease physical activity resulting in a vicious cycle of decreased activity and accelerated mobility decline. These changes may place individuals with PAD at greater risk for sarcopenia. Sarcopenia has a prevalence in other clinical populations of $\sim 1-22 \%$ and increases the risk for disability. This study sought to 1) determine the prevalence of sarcopenia in males with PAD and 2) compare the functional status of individuals with PAD with and without sarcopenia. Sixtythree participants with PAD (38\% African American, age: $70.1 \pm 0.7$ years, BMI: $28.1 \pm 0.5 \mathrm{~kg} / \mathrm{m}^{2}, \mathrm{ABI}: 0.65 \pm 0.01$ $\mathrm{X} \pm \mathrm{SEM})$ underwent DXA scans to assess appendicular lean mass (ALM), and performed a six-minute walk test to determine six-minute walk distance(6MWD) and a treadmill test to measure claudication onset time (COT) and peak walking time (PWT). Sarcopenia was defined as ALM/ $\mathrm{ht}^{2}$ of less than $7.4 \mathrm{~kg} / \mathrm{m}^{2}$. Independent t-tests were used to compare 6MWD, COT and PWT. Sarcopenia prevalence in our PAD cohort was $17 \%$. Individuals with sarcopenia $(\mathrm{N}=11)$ had lower $6 \mathrm{MWD}(\mathrm{p}<0.05 ; 345 \pm 21$ vs $392 \pm 10$ meters) and COT ( $<<0.05 ; 106 \pm 10$ vs $183 \pm 19$ seconds) compared to individuals without sarcopenia $(\mathrm{N}=52)$. There was no difference in PWT between the groups $(\mathrm{p}=0.4$; $345 \pm 21$ vs $392 \pm 10$ ) Men with PAD demonstrate a prevalence of sarcopenia consistent with other clinical populations and those with sarcopenia and PAD demonstrate decreased function.

\section{ASSOCIATION OF MIDLIFE HYPERTENSION WITH LATE-LIFE HEARING LOSS}

N. Reed, J.A. Deal, F. Lin, A. Sharrett, Johns Hopkins

University, Baltimore, Maryland

The contribution of hypertension to hearing impairment (HI) in older adults is poorly understood. Hypertension could plausibly contribute to impaired circulation in the cochlea and thereby disrupt metabolic activity related to hearing. However, results from previous epidemiologic studies of the association of hypertension and hearing loss have been inconsistent, and the relative importance of midlife versus late-life hypertension is unknown.

We analyzed data from 248 men and women (4564 years at baseline, 1987-1989) in the Atherosclerosis Risk in Communities (ARIC) Study to quantify the relationship of mid- and late-life hypertension with late-life HI. Blood pressure was measured over 5 visits that were interspersed from 1997-2013. Hypertension was defined as systolic blood pressure (SBP) $\geq 140 \mathrm{mmHg}$, diastolic blood pressure (DBP) $\geq 90 \mathrm{mmHg}$, or antihypertensive use. SBP and DBP were also modeled continuously. Hearing was assessed using pure tone audiometry in 2013. A better-hearing ear pure-tone average (PTA) in decibels hearing level (db HL) was calculated using thresholds from $0.5-4 \mathrm{kHz}$ and modeled continuously The difference in PTA associated with hypertension was estimated using multivariable linear regression.

47 participants $(19 \%)$ had hypertension at Visit 1(198789) compared to $183(74 \%)$ at Visit 5(2013). In analyses adjusted for demographic and clinical covariates, midlife SBP was associated with HI, but late-life SBP was not (difference in PTA per 10mm Hg SBP measured at Visit 1(1987-89): $1.43 \mathrm{~dB}$ HL $(95 \%$ CI: $0.32,2.53)$ vs. Visit 5(2013): $-0.43 \mathrm{~dB}$ HL (95\% CI: $-1.41,0.55)$.

Our results demonstrate that midlife, but not late-life, SBP was independently associated with poorer hearing.

\section{SELF RATED HEALTH IS ASSOCIATED WITH SERUM GLUCOSE AMONG ELDERLY LIVING AT RURAL AREA IN GUINEA}

Y. Kasahara ${ }^{1}$, M. Hirosaki ${ }^{2}$, Y. Ishimoto ${ }^{3}$, M. Nakatsuka ${ }^{4}$, M. Fujisawa ${ }^{4}$, K. Matsubayashi, 1. Shonan University of Medical Science, Yokohama, Japan, 2. Fukushima Medical University, Fukushima, Japan, 3. Mie University, Tsu, Japan, 4. Kyoto University, Kyoto, Japan

Aim: SRH is an important outcome measure along with mortality, morbidity, and clinical outcomes. To our knowledge, however, little is known about the association between SRH and comprehensive geriatric functions in Guinea. We examined the association between SRH and comprehensive geriatric functions in rural area in Guinea.

Methods: The study population consisted of 129 people aged 60 and older $(m e n=42$, women $=87$, mean age $68.59 \pm 7.5$ ) living in Bossou, Nzerekore prefecture, Guinea in 2009. They were screened by the self-reported questionnaire contain SRH, basic ADL, moods, life styles and medical conditions. Furthermore, medical doctor checked blood serum and hemoglobin simply. SRH was assessed with the following question, "How would you rate your current health status?" using a $100-\mathrm{mm}$ visual analogue scale (worst $=0$ to best $=100$ ). Mood was assessed with the following question, "Do you often feel discourage yourself?" Using the median SRH score of 43, we defined participants with a SRH score $>43$ as the high SRH group and those with a score $\leq 43$ as the low SRH group.

Result: Elderly people with low SRH had significantly higher glucose serum, lower scores for each ADL item, functional reach length and self-rated happiness compared to those with low SRH. 